\title{
Rule-Based Form for Stream Constraints
}

\author{
Kasper Dokter ${ }^{(凶)}$ and Farhad Arbab \\ Centrum Wiskunde \& Informatica, Amsterdam, Netherlands \\ K.P.C.Dokter@cwi.nl
}

\begin{abstract}
Constraint automata specify protocols as labeled transition systems that preserve synchronization under composition. They have been used as a basis for tools, such as compilers and model checkers. Unfortunately, composition of transition systems suffers from state space and transition space explosions, which limits scalability of the tools based on constraint automata. In this work, we propose stream constraints as an alternative to constraint automata that avoids state space explosions. We introduce a rule-based form for stream constraints that can avoid transition space explosions. We provide sufficient conditions under which our approach avoids transition space explosions.
\end{abstract}

\section{Introduction}

Over a decade ago, Baier et al. introduced constraint automata for the specification of interaction protocols [6]. Constraint automata feature a powerful composition operator that preserves synchrony: composite constructions not only yield intuitively meaningful asynchronous protocols but also synchronous protocols. Constraint automata have been used as basis for tools, like compilers and model checkers. Jongmans developed Lykos: a compiler that translates constraint automata into reasonably efficient executable Java code [13]. Baier, Blechmann, Klein, and Klüppelholz developed Vereofy, a model checker for constraint automata [4,19]. Unfortunately, like every automaton model, composition of constraint automata suffers from state space and transition space explosions. These explosions limit the scalability of the tools based on constraint automata.

To improve scalability, Clarke et al. developed a compiler that translates a constraint automaton to a first-order formula [9]. The transitions of the constraint automaton correspond to the solutions of this formula. At run time, a generic constraint solver finds these solutions and simulates the automaton. Since composition and abstraction for constraint automata respectively correspond to conjunction and existential quantification, the first-order specification does not suffer from state space or transition space explosion. However, the approach proposed by Clarke et al. only delays the complexity until run time: calling a generic constraint solver at run time imposes a significant overhead.

Jongmans realized that the overhead of this constraint solver is not always necessary. He developed a commandification algorithm that accepts constraints without disjunctions (i.e., conjunctions of literals) and translates them into a 
small imperative program [14]. The resulting program is a light-weight, tailormade constraint solver with minimal run time overhead. Since commandification accepts only constraints without disjunction, Jongmans applied this technique to data constraints on individual transitions in a constraint automaton. Relying on constraint automata, his approach still suffers from scalability issues [17].

We aim to prevent state space and transition space explosions by combining the ideas of Clarke et al. and Jongmans. To this end, we present the language of stream constraints: a generalization of constraint automata based on temporal logic. A stream constraint is an expression that relates streams of observed data at different locations (Sect. 2). We identify a subclass of stream constraints, called regular (stream) constraints, which is closed under composition and abstraction (Sect. 3). Regular constraints can be viewed as a constraint automata, and conjunction of reflexive regular constraints is similar to composition of constraint automata (Sect. 4).

A straightforward application of the commandification algorithm of Jongmans to regular stream constraints entails transforming a stream constraint into disjunctive normal form and applying the algorithm to each clause separately. However, the number of clauses in the disjunctive normal form may grow exponentially in the size of the composition. To prevent such exponential blowups of the size of the formula, we recognize and exploit symmetries in the disjunctive normal form. Each clause in the disjunctive normal form can be constructed from a set of basic stream constraints, which we call rules. This idea allows us to represent a single large constraint as certain combination of a set of smaller constraints, called the rule-based form (Sect. 5). We express the composition of stream constraints in terms of the rule-based normal form (Sect.6), and show that, for simple sets of rules, the number of rules to describe the composition is only linear in the size of the composition (Sect. 7). The class of stream constraints defined by a simple set of rules contains constraints for which the size of the disjunctive normal form explodes, which shows that our approach improves upon existing approaches by Clarke et al. and Jongmans. We express abstraction on stream constraints in terms of the rule-based normal form and provide a sufficient condition under which the number of rules remains constant (Sect.8). Finally, we conclude and point out future work (Sect.10).

Related work. Representation of stream constraints in rule-based form is part of a larger line of research on symbolic approaches, such a symbolic model checking $[5,8,20]$ and symbolic execution [10]. These approaches not only use logic (cf., SAT solving techniques $[12,18]$ for verification), but also other implicit representations, like binary decision diagrams [7] and Petri nets [21]. Petri nets offer a small representation of protocols with an exponentially large state space. While our focus is more on compilation, Petri nets have been studied in the context of verification. As inspiration for future work, it is interesting to study the similarities between Petri nets and stream constraints.

Since regular stream constraints correspond to constraint automata, we can view regular stream constrains as a restricted temporal logic for which distributed synthesis is easy. In general, distributed (finite state) synthesis of 
protocols is undecidable $[22,23]$. Pushing the boundary from regular to a larger class of stream constraints can be useful for more effective synthesis methods.

\section{Syntax and Semantics}

The semantics of constraint automata is defined as a relation over timed data streams [3], which are pairs, each consisting of a non-decreasing stream of time stamps and a stream of observed (exchanged) data items. The primary significance of time streams is the proper alignment of their respective data streams, by allowing "temporal gaps" during which no data is observed. For convenience, we drop the time stream and model protocols as relations over streams of data, augmented by a special symbol that designates "no-data" item.

We first define the abstract behavior of a protocol $C$. Fix an infinite set $X$ of variables, and fix a non-empty set of user-data Data $\supseteq\{0\}$ that contains a datum 0. Consider the data domain $D=D a t a \cup\{*\}$ of data stream items, where we use the "no-data" symbol $* \in D \backslash$ Data to denote the absence of data. We model the a single execution of protocol $C$ as a function

$$
\theta: X \longrightarrow D^{\mathbb{N}}
$$

that maps every variable $x \in X$ to a function $\theta(x): \mathbb{N} \longrightarrow D$ that represents a stream of data at location $x$. We call $\theta$ a data stream tuple (over $X$ and $D$ ). For all $n \in \mathbb{N}$ and all $x \in X$, the value $\theta(x)(n) \in D$ is the data that we observe at location $x$ and time step $n$. If $\theta(x)(n)=*$, we say that no data is observed at $x$ in step $n$ (i.e., we may view $\theta$ as a partial map $\mathbb{N} \times X \rightarrow$ Data). The behavior of protocol $C$ consists of the set

$$
\mathcal{L}(C) \subseteq\left(D^{\mathbb{N}}\right)^{X}
$$

of all possible executions of $C$, called the accepted language of $C$. We can think of accepted language $\mathcal{L}(C)$ as a relation over data streams. In this paper, we study protocols that are defined as a stream constraint:

Definition 1 (Stream constraints). A stream constraint $\phi$ is an expression generated by the following grammar

$$
\begin{aligned}
\phi & :=\perp\left|t_{0} \doteq t_{1}\right| \phi_{0} \wedge \phi_{1}|\neg \phi| \exists x \phi \mid \square \phi \\
t & ::=x|\boldsymbol{d}| t^{\prime}
\end{aligned}
$$

where $x \in X$ is a variable, $d \in D$ is a datum, and $t$ is a stream term.

We use the following standard syntactic sugar: $\top=\neg \perp, \phi_{0} \vee \phi_{1}=\neg\left(\neg \phi_{0} \wedge\right.$ $\left.\neg \phi_{1}\right), \diamond \phi=\neg \square \neg \phi, \neg\left(t_{1} \doteq t_{2}\right)=\left(t_{1} \neq t_{2}\right),\left(t_{1} \doteq t_{2} \wedge \cdots \wedge t_{n-1} \doteq t_{n}\right)=\left(t_{1} \doteq\right.$ $\left.\cdots \doteq t_{n}\right), t^{(0)}=t$, and $t^{(k+1)}=\left(t^{(k)}\right)^{\prime}$, for all $k \geq 0$. Following Rutten [25], we call $t^{(k)}, k \geq 0$, the $k$-th derivative of term $t$.

We interpret a stream constraint as a constraint over streams of data in $D^{\mathbb{N}}$. For a datum $d \in D, \boldsymbol{d}$ is the constant stream defined as $\boldsymbol{d}(n)=d$, for all $n \in \mathbb{N}$. The operator $(-)^{\prime}$, called stream derivative, drops the head of the stream and 
is defined as $\sigma^{\prime}(n)=\sigma(n+1)$, for all $n \in \mathbb{N}$ and $\sigma \in D^{\mathbb{N}}$. Streams can be related by $\doteq$ that expresses equality of their heads: $x \doteq y$ iff $x(0)=y(0)$, for all $x, y \in D^{\mathbb{N}}$. The modal operator $\square$ allows us to express that a stream constraint holds after applying any number of derivatives to all variables. For example, $\square(x \doteq y)$ iff $x^{(k)}(0)=y^{(k)}(0)$, for all $k \in \mathbb{N}$ and $x, y \in D^{\mathbb{N}}$. Stream constraints can be composed via conjunction $\wedge$, or negated via negation $\neg$. Streams can be hidden via existential quantification $\exists$.

Each stream term $t$ evaluates to a data stream in $D^{\mathbb{N}}$. Let $\theta: X \longrightarrow D^{\mathbb{N}}$ be a data stream tuple. We extend the domain of $\theta$ from the set of variables $X$ to the set of terms $T \supseteq X$ as follows: we define $\theta: T \longrightarrow D^{\mathbb{N}}$ via $\theta(\boldsymbol{d})=\boldsymbol{d}$ and $\theta\left(t^{\prime}\right)=\theta(t)^{\prime}$, for all $d \in D$ and terms $t \in T$.

Next, we interpret a stream constraint $\phi$ as a relation over streams.

Definition 2 (Semantics). The language $\mathcal{L}(\phi) \subseteq\left(D^{\mathbb{N}}\right)^{X}$ of a stream constraint $\phi$ over variables $X$ and data domain $D$ is defined as

1. $\mathcal{L}(\perp)=\emptyset$;

2. $\mathcal{L}\left(t_{0} \doteq t_{1}\right)=\left\{\theta: X \longrightarrow D^{\mathbb{N}} \mid \theta\left(t_{0}\right)(0)=\theta\left(t_{1}\right)(0)\right\}$;

3. $\mathcal{L}\left(\phi_{0} \wedge \phi_{1}\right)=\mathcal{L}\left(\phi_{0}\right) \cap \mathcal{L}\left(\phi_{1}\right)$;

4. $\mathcal{L}(\neg \phi)=\left(D^{\mathbb{N}}\right)^{X} \backslash \mathcal{L}(\phi) ;$

5. $\mathcal{L}(\exists x \phi)=\left\{\theta: X \longrightarrow D^{\mathbb{N}} \mid \theta[x \mapsto \sigma] \in \mathcal{L}(\phi)\right.$, for some $\left.\sigma \in D^{\mathbb{N}}\right\}$;

6. $\mathcal{L}(\square \phi)=\left\{\theta: X \longrightarrow D^{\mathbb{N}} \mid \theta^{(k)} \in \mathcal{L}(\phi)\right.$, for all $\left.k \geq 0\right\}$,

where $\theta[x \mapsto \sigma]: X \longrightarrow D^{\mathbb{N}}$ is defined as $\theta[x \mapsto \sigma](x)=\sigma$ and $\theta[x \mapsto \sigma](y)=$ $\theta(y)$, for all $y \in X \backslash\{x\}$; and $\theta^{(k)}: X \longrightarrow D^{\mathbb{N}}$ is defined as $\theta^{(k)}(x)=\theta\left(x^{(k)}\right)$, for all $x \in X$.

Let $\phi$ and $\psi$ be two stream constraints and $\theta: X \longrightarrow D^{\mathbb{N}}$ a data stream tuple. We say that $\theta$ satisfies $\phi$ (and write $\theta \models \phi$ ), whenever $\theta \in \mathcal{L}(\phi)$. We say that $\phi$ implies $\psi$ (and write $\phi \models \psi$ ), whenever $\mathcal{L}(\phi) \subseteq \mathcal{L}(\psi)$. We call $\phi$ and $\psi$ equivalent (and write $\phi \equiv \psi$ ), whenever $\mathcal{L}(\phi)=\mathcal{L}(\psi)$.

Example 1. One of the simplest stream constraints is $\operatorname{sync}(a, b)$, which is defined as $\square(a \doteq b)$. Constraint $\operatorname{sync}(a, b)$ encodes that the data streams at $a$ and $b$ are equal: $\theta(a)(k)=\theta(b)(k)$, for all $k \in \mathbb{N}$ and all $\theta \in\left(D^{\mathbb{N}}\right)^{X}$. Therefore, $\operatorname{sync}(a, b)$ synchronizes the data flow observed at ports $a$ and $b$.

Conjunction $\wedge$ and existential quantification $\exists$ provide natural operators for composition and abstraction for stream constraints. For example, the composition $\operatorname{sync}(a, b) \wedge \operatorname{sync}(b, c)$ synchronizes ports $a, b$, and $c$. Hiding port $b$ yields $\exists b(\operatorname{sync}(a, b) \wedge \operatorname{sync}(b, c))$, which is equivalent to $\operatorname{sync}(a, c)$.

Example 2. Recall that $x^{(k)}$, for $k \geq 0$, is the $k$-th derivative of $x$. We can express that a stream $x$ is periodic via the stream constraint $\square\left(x^{(k)} \doteq x\right)$, for some $k \geq 1$. For $k=1$, stream $x$ is constant, like $\mathbf{0}$ and $*$.

Example 3. The stream constraint fifo $(a, b, m)$ defined as $m \doteq * \wedge \square\left(\left(a \doteq m^{\prime} \doteq\right.\right.$ $\left.\mathbf{0} \wedge b \doteq m \doteq *) \vee\left(a \doteq m^{\prime} \doteq * \wedge b \doteq m \doteq \mathbf{0}\right) \vee\left(a \doteq b \doteq * \wedge m^{\prime} \doteq m\right)\right)$ models a 1-place buffer with input location $a$, output location $b$, and memory location $m$ that can be full $(m \doteq \mathbf{0})$ or empty $(m \doteq *)$. 
Example 4. Recall that $*$ models absence of data. Stream constraint $\square \diamond(a \neq *)$ expresses that always eventually we observe some datum at $a$. A constraint of such form can be used to define fairness.

\section{Regular Constraints}

We identify a subclass of stream constraints that naturally correspond to constraint automata. We first introduce some notation.

To denote that a string $s$ occurs as a substring in a stream constraint $\phi$ or a stream term $t$, we write $s \in \phi$ or $s \in t$, respectively.

Every stream constraint $\phi$ admits a set free $(\phi) \subseteq X$ of free variables, defined inductively via free $(\perp)=\emptyset$, free $\left(t_{0} \doteq t_{1}\right)=\left\{x \in X \mid x \in t_{0}\right.$ or $\left.x \in t_{1}\right\}$, free $\left(\phi_{0} \wedge \phi_{1}\right)=$ free $\left(\phi_{0}\right) \cup$ free $\left(\phi_{1}\right)$, free $(\neg \phi)=$ free $(\square \phi)=$ free $(\phi)$, and free $(\exists x \phi)=$ free $(\phi) \backslash\{x\}$.

For every variable $x \in X$, we define the degree of $x$ in $\phi$ as

$$
\operatorname{deg}_{x}(\phi)=\max \left(\{-1\} \cup\left\{k \geq 0 \mid x^{(k)} \in \phi\right\}\right),
$$

and the degree of $\phi$ as $\operatorname{deg}(\phi)=\max _{x \in X} \operatorname{deg}_{x}(\phi)$. Note that for $x \notin \phi$ we have $\operatorname{deg}_{x}(\phi)=-1$. For $k \geq 0$, we write $\operatorname{free}^{k}(\phi)=\left\{x \in\right.$ free $\left.(\phi) \mid \operatorname{deg}_{x}(\phi)=k\right\}$ for the set of all free variables of $\phi$ of degree $k$.

We call a variable $x$ of degree zero in $\phi$ a port variable and write $P(\phi)=$ free $^{0}(\phi)$ for the set of port variables of $\phi$. We call a variable $x$ of degree one or higher in $\phi$ a memory variable and write $M(\phi)=\bigcup_{k \geq 1}$ free $^{k}(\phi)$ for the set of memory variables of $\phi$.

Definition 3 (Regular). A stream constraint $\phi$ is regular if and only if $\phi=\psi_{0} \wedge \square \psi$, such that $\square \notin \psi_{0} \wedge \psi$ and $\operatorname{deg}_{x}\left(\psi_{0}\right)<\operatorname{deg}_{x}(\psi) \leq 1$, for all $x \in X$.

For a regular stream constraint $\phi=\psi_{0} \wedge \square \psi$, we refer to $\psi_{0}$ as the initial condition of $\phi$ and we refer to $\psi$ as the invariant of $\phi$. Stream constraints $\operatorname{sync}(a, b)$ and fifo $(a, b, m)$ in Examples 1 and 3 are regular stream constraints.

A regular stream constraint $\phi$ has an operational interpretation in terms of a labeled transition system $\llbracket \phi \rrbracket$. States of the transition system consist of maps $q: M(\phi) \longrightarrow D$ that assign data to memory locations, and its labels consist of maps $\alpha: P(\phi) \longrightarrow D$ that assign data to ports. We write $Q(\phi)$ for the set of states of $\phi$ and $A(\phi)$ for the set of labels of $\phi$.

Definition 4 (Operational semantics). The operational semantics $\llbracket \rrbracket$ of a regular stream constraint $\phi=\psi_{0} \wedge \square \psi$ consists of a labeled transition system $\left(Q(\phi), A(\phi), \rightarrow, Q_{0}\right)$, with set of states $Q(\phi)$, set of labels $A(\phi)$, set of transitions $\rightarrow=\left\{\left(q_{\phi}(\theta), q_{\phi}\left(\theta^{\prime}\right), \alpha_{\phi}(\theta)\right) \mid \theta \in \mathcal{L}(\psi)\right\}$, and set of initial states $Q_{0}=\left\{q_{\phi}(\theta) \mid\right.$ $\left.\theta \in \mathcal{L}\left(\psi_{0} \wedge \psi\right)\right\}$, where

1. $q_{\phi}(\theta): M(\phi) \longrightarrow D$ is defined as $q_{\phi}(\theta)(x)=\theta(x)(0)$, for $x \in M(\phi)$; and

2. $\alpha_{\phi}(\theta): P(\phi) \longrightarrow D$ is defined as $\alpha_{\phi}(\theta)(x)=\theta(x)(0)$, for $x \in P(\phi)$.

and $\theta^{\prime}$ is defined as $\theta^{\prime}(x)(n)=\theta(x)(n+1)$, for all $x \in X$ and $n \in \mathbb{N}$. 


$$
[a \mapsto *, b \mapsto *]
$$

Fig. 1. Semantics of fifo $(a, b, m)$ over the trivial data domain $\{0, *\}$.

Example 5. Consider the regular stream constraint fifo $(a, b, m)$ from Example 3. Note that in this example, the set of ports equals free ${ }^{0}$ (fifo) $=\{a, b\}$ and the set of memory locations equals free ${ }^{1}$ (fifo) $=\{m\}$. The semantics of fifo $(a, b, m)$ over the trivial data domain $D=\{0, *\}$ consists of 4 transitions:

1. $([m \mapsto *],[m \mapsto 0],[a \mapsto 0, b \mapsto *])$;

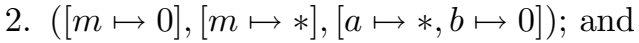

3. ( $[m \mapsto d],[m \mapsto d],[a \mapsto *, b \mapsto *])$, for every $d \in\{*, 0\}$.

Figure 1 shows the semantics of fifo over the trivial data domain.

Equivalent stream constraints do not necessarily have the same operational semantics. We are, therefore, interested in operational equivalence of constraints:

Definition 5 (Operational equivalence). Stream constraints $\phi$ and $\psi$ are operationally equivalent $(\phi \simeq \psi)$ iff $\phi \equiv \psi$ and $\operatorname{free}^{k}(\phi)=\operatorname{free}^{k}(\psi)$, for $k \geq 0$.

Example 6. Let $\phi$ be a stream constraint, let $t$ be a term and let $x \notin t$ be a variable that does not occur in $t$. Then, we have $\exists x(x \doteq t \wedge \phi) \equiv \phi[t / x]$, where $\phi[t / x]$ is obtained from $\phi$ by substituting $t$ for every free occurrence of $x$. Observe that $\exists x(x \doteq t \wedge \phi)$ and $\phi[t / x]$ may admit different sets of free variables: if $\phi$ is just $\top$ and $t$ is a variable $y$, the equivalence amounts to $\exists x(x \doteq y) \equiv \top$. To ensure that the free variables coincide, we can add the equality $t \doteq t$ and obtain the operational equivalence $\exists x(x \doteq t \wedge \phi) \simeq \phi[t / x] \wedge t \doteq t$.

Operational equivalence of stream constraints $\phi$ and $\psi$ implies that their operational semantics are identical, i.e., $\llbracket \phi \rrbracket=\llbracket \psi \rrbracket$. It is possible to introduce weaker equivalences by, for example, demanding that $\llbracket \phi \rrbracket$ and $\llbracket \psi \rrbracket$ are only weakly bisimilar. Such weaker equivalence offer more room for simplification of stream constraints than operational equivalence does. As our work does not need this generality, we leave the study of such weaker equivalences as future work.

The most important operations on stream constraints are composition $(\wedge)$ and hiding $(\exists)$. The following result shows that regular stream constraints are closed under conjunction and existential quantification of degree zero variables.

Theorem 1. For all stream constraints $\phi$ and $\psi$ and variables $x$, we have

1. $\square \phi \wedge \square \psi \equiv \square(\phi \wedge \psi)$; and

2. $\exists x \square \phi \equiv \square \exists x \phi$, whenever $\operatorname{deg}_{x}(\phi) \leq 0$ and $\square \notin \phi$. 
Proof. For assertion 1, $\mathcal{L}(\square \phi \wedge \square \psi)=\left\{\theta \in\left(D^{\mathbb{N}}\right)^{X}\left|\forall k \geq 0: \theta^{(k)}\right|=\phi \wedge \psi\right\}=$ $\mathcal{L}(\square(\phi \wedge \psi))$ shows that $\square \phi \wedge \square \psi \equiv \square(\phi \wedge \psi)$.

For assertion 2, suppose that $\operatorname{deg}_{x}(\phi) \leq 0$ and $\square \notin \phi$. We show that $\theta \in$ $\mathcal{L}(\square \exists x \phi)$ if and only if $\theta \in \mathcal{L}(\exists x \square \phi)$, for all $\theta \in\left(D^{\mathbb{N}}\right)^{X}$. By Definition 2, this equivalence can be written as

$$
\theta^{(k)}\left[x \mapsto \mu_{k}\right] \models \phi \quad \Leftrightarrow \quad(\theta[x \mapsto \sigma])^{(k)} \models \phi,
$$

for all $k \geq 0, \sigma \in D^{\mathbb{N}}$, and $\mu_{k} \in D^{\mathbb{N}}$ such that $\mu_{k}(0)=\sigma^{(k)}(0)$.

To prove Eq. (3), we proceed by induction on the length of $\phi$ :

Case $1(\phi:=\perp)$ : Since $\mathcal{L}(\perp)=\emptyset$, Eq. (3) holds trivially.

Case $2\left(\phi:=t_{0} \doteq t_{1}\right)$ : Observe that, since $\operatorname{deg}_{x}(\phi) \leq 0$, for all terms $t$, we have $x \in t$ iff $t=x$. We conclude Eq. (3) from $\mu_{k}(0)=\sigma^{(k)}(0)$ and

$$
\theta^{(k)}\left[x \mapsto \mu_{k}\right](t)(0)=\left\{\begin{array}{ll}
\mu_{k}(0) & \text { if } t=x \\
\theta^{(k)}(t)(0) & \text { if } t \neq x
\end{array}\right\}=(\theta[x \mapsto \sigma])^{(k)}(t)(0) .
$$

Case $3\left(\phi:=\psi_{0} \wedge \psi_{1}\right)$ : By the induction hypothesis, Eq. (3) holds for $\psi_{0}$ and $\psi_{1}$. By conjunction of Eq. (3), we conclude Eq. (3) for $\phi$.

Case $4(\phi:=\neg \psi)$ : By the induction hypothesis, Eq. (3) holds for $\psi$. By contraposition of Eq. (3), we conclude Eq. (3) for $\phi$.

Case $5(\phi:=\exists y \psi)$ : If $y=x$, then $x \notin$ free $(\phi)$ and both sides in Eq. (3) are equivalent to $\theta^{(k)}=\phi$. Hence, Eq. (3) holds for $y=x$. Suppose $y \neq x$. Then, $\theta^{(k)}\left[x \mapsto \mu_{k}\right] \models \phi$ is equivalent to $(\theta[y \mapsto \tau])^{(k)}\left[x \mapsto \mu_{k}\right] \models \psi$, for some $\tau \in D^{\mathbb{N}}$. Applying the induction hypothesis for $\theta$ equal to $\theta[y \mapsto \tau]$, we conclude that $\theta^{(k)}\left[x \mapsto \mu_{k}\right] \models \phi$ is equivalent to $(\theta[y \mapsto \tau][x \mapsto \sigma])^{(k)} \models \psi$, for some $\tau \in D^{\mathbb{N}}$. Since $y \neq x$, we conclude that Eq. (3) holds.

We conclude that the claim holds for all $\phi$ with $\operatorname{deg}_{x}(\phi) \leq 0$ and $\square \notin \phi$.

\section{Reflexive Constraints}

Conjunction of stream constraints is a simple syntactic composition operator with clear semantics: a data stream tuple $\theta$ satisfies a conjunction $\phi_{0} \wedge \phi_{1}$ if and only if $\theta$ satisfies both $\phi_{0}$ and $\phi_{1}$. In view of the semantics of regular stream constraints in Definition 2, it is less obvious how $\llbracket \phi_{0} \wedge \phi_{1} \rrbracket$ relates to $\llbracket \phi_{0} \rrbracket$ and $\llbracket \phi_{1} \rrbracket$. The following result characterizes their relation when no memory is shared.

Theorem 2. Let $\phi_{0}$ and $\phi_{1}$ be regular stream constraints such that free $\left(\phi_{0}\right) \cap$ free $\left(\phi_{1}\right) \subseteq P\left(\phi_{0} \wedge \phi_{1}\right)$, and let $\left(q_{i}, q_{i}^{\prime}, \alpha_{i}\right) \in Q\left(\phi_{i}\right)^{2} \times A\left(\phi_{i}\right)$, for $i \in\{0,1\}$. The following are equivalent:

1. $q_{0} \stackrel{\alpha_{0}}{\longrightarrow} q_{0}^{\prime}$ in $\llbracket \phi_{0} \rrbracket, q_{1} \stackrel{\alpha_{1}}{\longrightarrow} q_{1}^{\prime}$ in $\llbracket \phi_{1} \rrbracket$, and $\left.\alpha_{0}\right|_{P\left(\phi_{1}\right)}=\left.\alpha_{1}\right|_{P\left(\phi_{0}\right)}$;

2. $q_{0} \cup q_{1} \stackrel{\alpha_{0} \cup \alpha_{1}}{\longrightarrow} q_{0}^{\prime} \cup q_{1}^{\prime}$ in $\llbracket \phi_{0} \wedge \phi_{1} \rrbracket$,

where $\mid$ is restriction of maps, and $\cup$ is union of maps. 
Proof. Write $\phi_{i}=\psi_{i 0} \wedge \square \psi_{i}$, with $\square \notin \psi_{i 0} \wedge \psi_{i}$ and $\operatorname{deg}_{x}\left(\psi_{i 0}\right)<\operatorname{deg}_{x}\left(\psi_{i}\right) \leq 1$, for all $x \in X$. Then, free ${ }^{k}\left(\phi_{i}\right)=$ free $^{k}\left(\psi_{i}\right)$, for all $i, k \in\{0,1\}$.

Suppose that assertion 1 holds. By Definition 2, we find, for all $i \in\{0,1\}$, some $\theta_{i} \in \mathcal{L}\left(\psi_{i}\right)$ such that $q_{i}=q_{\phi_{i}}\left(\theta_{i}\right), q_{i}^{\prime}=q_{\phi_{i}}\left(\theta_{i}^{\prime}\right)$, and $\alpha_{i}=\alpha_{\phi_{i}}\left(\theta_{i}\right)$. Define $\theta: X \longrightarrow D^{\mathbb{N}}$ by $\theta(x)=\theta_{i}(x)$, if $x \in$ free $\left(\phi_{i}\right)$, and $\theta(x)=*$, otherwise. Since free $\left(\phi_{0}\right) \cap \operatorname{free}\left(\phi_{1}\right) \subseteq P\left(\phi_{0} \wedge \phi_{1}\right)$ and $\left.\alpha_{0}\right|_{P\left(\phi_{1}\right)}=\left.\alpha_{1}\right|_{P\left(\phi_{0}\right)}$, we have that $\theta_{0}(x)=$ $\theta_{1}(x)$, for all $x \in$ free $\left(\phi_{0}\right) \cap$ free $\left(\phi_{1}\right)$. Hence, $\theta$ is well-defined. By construction, $\theta \models \psi_{0}$ and $\theta \models \psi_{1}$. By Definition 2, we have $\theta \models \psi_{0} \wedge \psi_{1}$. By Theorem 1 , we have $\phi_{0} \wedge \phi_{1}=\psi_{00} \wedge \psi_{10} \wedge \square\left(\psi_{0} \wedge \psi_{1}\right)$. Since $q_{0} \cup q_{1}=q_{\phi_{0} \wedge \phi_{1}}(\theta), q_{0}^{\prime} \cup q_{1}^{\prime}=q_{\phi_{0} \wedge \phi_{1}}\left(\theta^{\prime}\right)$, and $\alpha_{0} \cup \alpha_{1}=\alpha_{\phi_{0} \wedge \phi_{1}}(\theta)$, we conclude assertion 2 .

Suppose that assertion 2 holds. We find some $\theta \in \mathcal{L}\left(\psi_{0} \wedge \psi_{1}\right)$, such that $q_{0} \cup q_{1}=q_{\theta}, q_{0}^{\prime} \cup q_{1}^{\prime}=q_{\theta^{\prime}}$, and $\alpha_{0} \cup \alpha_{1}=\alpha_{\theta}$. Then, we conclude assertion 1, for $q_{i}=q_{\phi_{i}}(\theta), q_{i}^{\prime}=q_{\phi_{i}}\left(\theta^{\prime}\right)$, and $\alpha_{i}=\alpha_{\phi_{i}}(\theta)$.

Stream constraints $\phi_{0}$ and $\phi_{1}$ without shared variables $\left(\operatorname{free}\left(\phi_{0}\right) \cap \operatorname{free}\left(\phi_{1}\right)=\right.$ $\emptyset)$ seem completely independent. However, Theorem 2 shows that their composition $\phi_{0} \wedge \phi_{1}$ admits a transition only if $\phi_{0}$ and $\phi_{1}$ admit respective local transitions $\left(q_{0}, q_{0}^{\prime}, \alpha_{0}\right)$ and $\left(q_{1}, q_{1}^{\prime}, \alpha_{1}\right)$, such that $\left.\alpha_{0}\right|_{P\left(\phi_{1}\right)}=\left.\alpha_{1}\right|_{P\left(\phi_{0}\right)}$. Since $\phi_{0}$ and $\phi_{1}$ do not share variables, the latter condition on $\alpha_{0}$ and $\alpha_{1}$ is trivially satisfied. Still, for one protocol $\phi_{i}$, with $i \in\{0,1\}$, to make progress in the composition $\phi_{0} \wedge \phi_{1}$, constraint $\phi_{1-i}$ must admit an idling transition.

To allow such independent progress, we assume that $\phi_{1-i}$ admits an idling transition $(q, q, \tau)$, where $\tau$ is the silent label over $P\left(\phi_{1-i}\right)$. The silent label over a set of ports $P \subseteq X$ is the map $\tau: P \longrightarrow D$ that maps $x \in P$ to $* \in D$. If such idling transitions are available in every state of $\phi_{1}$, we say that $\phi_{1}$ is reflexive:

Definition 6 (Reflexive). A stream constraint $\phi$ is reflexive if and only if $q \stackrel{\tau}{\rightarrow} q$ in $\llbracket \phi \rrbracket$, for all $q \in Q(\phi)$.

For regular constraints, we can define reflexiveness also syntactically, for which we need some notation. For a variable $x \in X$ and an integer $k \in \mathbb{N} \cup\{-1\}$, we define the predicate $x \dagger_{k}$ (pronounced: " $x$ is blocked at step $k$ ") as follows:

$$
x \dagger_{k}:=\left(x^{(k)} \doteq x^{(k-1)}\right), \quad \text { with } x^{(k)} \doteq * \text {, for all } k<0 .
$$

Predicate $x \dagger_{-1} \equiv \top$ is trivially true. Predicate $x \dagger_{0} \equiv(x \doteq *)$ means that we observe no data flow at port $x$. Predicate $x \dagger_{1} \equiv\left(x^{\prime} \doteq x\right)$ means that the data in memory variable $x$ remains the same.

We now provide a syntactic equivalent of Definition 6 for regular constraints.

Lemma 1. A regular stream constraint $\phi=\psi_{0} \wedge \square \psi$ is reflexive if and only if $\bigwedge_{x \in X} x \dagger_{d(x)}=\psi$, where $d(x)=\operatorname{deg}_{x}(\phi)$, for all $x \in X$.

Proof. Since $d(x)=-1$, for all but finitely many $x \in X$, the stream constraint $\bigwedge_{x \in X} x \dagger_{d(x)}$ is well-defined. By definition, $\bigwedge_{x \in X} x \dagger_{d(x)} \models \psi$ if and only if, for all $q \in Q(\phi)$, there exists some $\theta \in \mathcal{L}(\psi)$, such that $q_{\theta}=q_{\theta^{\prime}}=q$ and $\alpha_{\theta}=\tau$. 
Example 7. The stream constraint $\operatorname{sync}(a, b):=\square(a \doteq b)$ from Example 1 is reflexive, because $\bigwedge_{x \in X} x \dagger_{d(x)}=a \doteq * \wedge b \doteq *$ implies $a \doteq b$. The stream constraint fifo from Example 3 is reflexive, because $\bigwedge_{x \in X} x \dagger_{d(x)}=a \doteq * \wedge b \doteq$ $* \wedge m^{\prime} \doteq m$ is one of the clauses of fifo.

Theorem 2 suggests a composition operator $\times$ on labeled transition systems, satisfying $\llbracket \phi_{0} \rrbracket \times \llbracket \phi_{1} \rrbracket=\llbracket \phi_{0} \wedge \phi_{1} \rrbracket$. For reflexive constraints $\phi_{0}$ and $\phi_{1}$, composition $\times$ simulates composition of constraint automata [6]. Constraint automata also feature a hiding operator that naturally corresponds to existential quantification $\exists$ for stream constraints. We leave a full formal comparison between stream constraints and constraint automata as future work.

\section{Rule-Based Form}

The commandification algorithm developed by Jongmans accepts only conjunctions of literals [14]. To apply commandification to the invariant $\psi$ of an arbitrary regular stream constraint $\psi_{0} \wedge \square \psi$, we can first transform $\psi$ into disjunctive normal form (DNF). However, the number of clauses in the disjunctive normal form may be exponential in the length of the constraint. In this section, we introduce an alternative to the disjunctive normal form that prevents such exponential blow up, for a strictly larger class of stream constraints. Our main observation is that the clauses of the disjunctive normal form may contain many symmetries, in the sense that we may generate all clauses from a set of stream constraints $R$, called a set of rules. A rule is a stream constraint $\rho$, such that $\operatorname{deg}(\rho) \leq 1$ and $\square \notin \rho$.

Definition 7 (Rule-based form). A reflexive stream constraint $\phi$ is in rulebased form iff $\phi$ equals

$$
\operatorname{rbf}(R)=\bigwedge_{x \in \operatorname{free}(R)} x \dagger_{d(x)} \vee \bigvee_{\rho \in R: x \in \operatorname{free}(\rho)} \rho
$$

with $R$ a finite set of rules, free $(R)=\bigcup_{\rho \in R}$ free $(\rho)$, and $d(x)=\max _{\rho \in R} \operatorname{deg}_{x}(\rho)$. $A$ stream constraint $\phi$ is defined by $R$ iff $\phi \simeq \operatorname{rbf}(R)$.

We apply the rule-based form to the invariant of regular constraints, via $\psi_{0} \wedge \square \operatorname{rbf}(R)$, for some degree zero stream constraint $\psi_{0}$ and set of rules $R$. Intuitively, $R$ remains smaller than the $\mathrm{DNF}$ of $\operatorname{rbf}(R)$ under composition.

Example 8. $\psi \simeq \operatorname{rbf}(\{\psi\})$, for all reflexive stream constraints $\psi$, with $\operatorname{deg}(\psi) \leq 1$ and $\square \notin \psi$. Hence, Example 7 shows sync $(a, b)=\square(a \doteq b) \simeq \square \operatorname{rbf}(\{a \doteq b\}) . \triangle$

Example 9. The stream constraint $\operatorname{lossy}(a, b):=\square \operatorname{rbf}(\{a \doteq a, a \doteq b\})$ is equivalent to $\square(b \doteq * \vee a \doteq b)$. Note that $\square \operatorname{rbf}(\{\top, a \doteq b\}) \simeq \square \operatorname{rbf}(\{a \doteq b\}) \simeq$ $\operatorname{sync}(a, b)$. Hence, rules $a \doteq a$ and $T$ are very different. 
Example 10. The set of rules that define a stream constraint is not unique. Consider the stream constraint fifo from Example 3. On the one hand, we have fifo $(a, b, m) \simeq m \doteq * \wedge \square \operatorname{rbf}(\{\varphi, \psi\})$, where $\varphi \simeq a \doteq m^{\prime} \doteq \mathbf{0} \wedge m \doteq *$ models the action that puts data in the buffer and $\psi \simeq m^{\prime} \doteq * \wedge b \doteq m \doteq \mathbf{0}$ models the action that takes data out of the buffer. On the other hand, we have fifo $(a, b, m) \simeq m \doteq$ $* \wedge \square \operatorname{rbf}\left(\left\{a \doteq m^{\prime} \doteq \mathbf{0} \wedge b \doteq m \doteq *, a \doteq m^{\prime} \doteq * \wedge b \doteq m \doteq \mathbf{0}\right\}\right)$.

Example 11. Rule-based forms are an alternative to disjunctive normal forms. Consider the reflexive constraint $\phi:=\bigvee_{i=1}^{n} \rho_{i}$ in DNF for which the first conjunctive clause $\rho_{1}$ is equivalent to $\bigwedge_{x \in \text { free }(\phi)} x \dagger_{d(x)}$, with $d(x)=\operatorname{deg}_{x}(\phi)$. By adding equalities of the form $x \doteq x$, we assume without loss of generality that free $\left(\rho_{i}\right)=$ free $(\phi)$, for all $2 \leq i \leq n$. For $R=\left\{\rho_{i} \mid 2 \leq i \leq n\right\}$, it follows from

$$
\operatorname{rbf}(R) \equiv \bigwedge_{x \in \text { free }(R)}\left(x \dagger_{d(x)} \vee \bigvee_{\rho \in R} \rho\right) \equiv\left(\bigwedge_{x \in \text { free }(\phi)} x \dagger_{d(x)}\right) \vee \bigvee_{\rho \in R} \rho \equiv \phi
$$

that $\phi$ is defined by the set $R$.

Definition 7 presents the rule-based form as a conjunctive normal form. The following result computes the disjunctive normal form of $\operatorname{rbf}(R)$.

Lemma 2. For every set of rules $R$, we have

$$
\operatorname{rbf}(R) \simeq \operatorname{dnf}(R):=\bigvee_{T \subseteq R} \bigwedge_{\rho \in T} \rho \wedge \bigwedge_{x \in \operatorname{free}(R) \backslash \operatorname{free}(T)} x \dagger_{d(x)} .
$$

Proof. Let $x \in X$ be arbitrary. By construction, we have $\operatorname{deg}_{x}(\operatorname{dnf}(R)) \leq$ $\max _{\rho \in R} \operatorname{deg}_{x}(\rho)$. Since $d(x)=\max _{\rho \in R} \operatorname{deg}_{x}(\rho)$, the clause for $T=\emptyset$ shows that $\operatorname{deg}_{x}(\operatorname{dnf}(R)) \geq d(x)$. By Lemma $4, \operatorname{deg}_{x}(\operatorname{rbf}(R))=\operatorname{deg}_{x}(\operatorname{dnf}(R))$, for all $x \in X$. Hence, free ${ }^{k}(\operatorname{rbf}(R))=$ free $^{k}(\operatorname{dnf}(R))$, for all $k \geq 0$.

Next, we show that $\operatorname{rbf}(R)=\operatorname{dnf}(R)$. Let $\theta \in \mathcal{L}(\operatorname{rbf}(R))$. We find, for every $x \in$ free $(R)$, some rule $\rho_{x} \in R$, such that $\theta=\rho$ and $x \in$ free $(\rho)$. Now, define $T_{\theta}:=$ $\left\{\rho_{x} \mid x \in\right.$ free $(R)$ and $\left.\theta \notin \mathcal{L}\left(x \dagger_{d(x)}\right)\right\}$. By construction, $\theta=\rho_{x}$, for every $\rho_{x} \in T_{\theta}$. If $x \in$ free $(R)$ and $\theta \notin \mathcal{L}\left(x \dagger_{d(x)}\right)$, then $\rho_{x} \in T_{\theta}$ and $x \in$ free $\left(\rho_{x}\right) \subseteq$ free $\left(T_{\theta}\right)$. By contraposition, we conclude that $\theta=x \dagger_{d(x)}$, for all $x \in$ free $(R) \backslash$ free $\left(T_{\theta}\right)$. Hence, $\theta=\operatorname{dnf}(R)$, and $\mathcal{L}(\operatorname{rbf}(R)) \subseteq \mathcal{L}(\operatorname{dnf}(R))$.

Finally, we show that $\operatorname{dnf}(R) \models \operatorname{rbf}(R)$. Let $\theta \in \mathcal{L}(\operatorname{dnf}(R))$. By definition of $\operatorname{dnf}(R)$, we find some $T \subseteq R$ with $\theta \models \rho$, for all $\rho \in T$, and $\theta \models x \dagger_{d(x)}$, for all $x \in$ free $(R) \backslash$ free $(T)$. Suppose that $x \in$ free $(R)$ and $\theta \not \models x \dagger_{d(x)}$. Since $\theta \models x \dagger_{d(x)}$, for all $x \in$ free $(R) \backslash$ free $(T)$, we find by contraposition that $x \in$ free $(T)$. Hence, we find some $\psi \in T$ with $x \in$ free $(\psi)$. Since $\theta \models \rho$, for all $\rho \in T$, we find that $\theta=\psi$. Hence, $\theta \models \operatorname{rbf}(R)$ and we conclude that $\operatorname{rbf}(R) \simeq \operatorname{dnf}(R)$.

\section{Composition}

We express conjunction of stream constraints in terms of their defining sets of rules. That is, for two sets of rules $R_{0}$ and $R_{1}$, we define the composition $R_{0} \wedge R_{1}$ 
of $R_{0}$ and $R_{1}$, such that $\operatorname{rbf}\left(R_{0} \wedge R_{1}\right) \simeq \operatorname{rbf}\left(R_{0}\right) \wedge \operatorname{rbf}\left(R_{1}\right)$. If $R_{0}$ and $R_{1}$ do not share any variable (i.e., free $\left(R_{0}\right) \cap$ free $\left(R_{1}\right)=\emptyset$ ), composition $R_{0} \wedge R_{1}$ is given by the union $R_{0} \cup R_{1}$. In this section, we define the composition $R_{0} \wedge R_{1}$ of $R_{0}$ and $R_{1}$ for free $\left(R_{0}\right) \cap$ free $\left(R_{1}\right) \neq \emptyset$.

In view of Example 11, consider the normal form $\operatorname{dnf}\left(R_{0} \wedge R_{1}\right)$. Since $\operatorname{dnf}\left(R_{0} \wedge\right.$ $R_{1}$ ) equals $\operatorname{dnf}\left(R_{0}\right) \wedge \operatorname{dnf}\left(R_{1}\right)$, it suffices to characterize the set of clauses of $\operatorname{dnf}\left(R_{0}\right) \wedge \operatorname{dnf}\left(R_{1}\right)$. Every such clause is a conjunction of a clause in $\operatorname{dnf}\left(R_{0}\right)$ and a clause in $\operatorname{dnf}\left(R_{1}\right)$. Lemma 2 shows that the clauses of $\operatorname{dnf}\left(R_{i}\right)$ correspond to subsets $T_{i}$ of $R_{i}$, for all $i \in\{0,1\}$. Not every pair of subsets $T_{0} \subseteq R_{0}$ and $T_{1} \subseteq R_{1}$ yields a clause of $\operatorname{dnf}\left(R_{0}\right) \wedge \operatorname{dnf}\left(R_{1}\right)$, but only if $S=T_{0} \cup T_{1}$ is synchronous:

Definition 8 (Synchronous). A synchronous set over sets of rules $R_{0}$ and $R_{1}$ is a subset $S \subseteq R_{0} \cup R_{1}$, with free $(S) \cap$ free $\left(R_{i}\right) \subseteq$ free $\left(S \cap R_{i}\right)$, for all $i \in\{0,1\}$.

Example 12. For any integer $i \geq 1$, let $\varphi_{i}:=a_{i} \doteq m_{i}^{\prime} \doteq \mathbf{0} \wedge m_{i} \doteq *$ and $\psi_{i}:=m_{i}^{\prime} \doteq * \wedge a_{i+1} \doteq m_{i} \doteq \mathbf{0}$ be the two rules that define fifo $\left(a_{i}, a_{i+1}, m_{i}\right)$, from Example 10. The synchronous sets consist of exactly those sets $S \subseteq\left\{\varphi_{1}, \psi_{1}\right\} \cup$ $\left\{\varphi_{2}, \psi_{2}\right\}$ that satisfy $\psi_{1} \in S$ iff $\varphi_{2} \in S$. That is, the synchronous sets are given by $\emptyset,\left\{\varphi_{1}\right\},\left\{\psi_{2}\right\},\left\{\psi_{1}, \varphi_{2}\right\},\left\{\varphi_{1}, \psi_{1}, \varphi_{2}\right\},\left\{\psi_{1}, \varphi_{2}, \psi_{2}\right\},\left\{\varphi_{1}, \psi_{1}, \varphi_{2}, \psi_{2}\right\}$.

Next, we recognize symmetries in the collection of synchronous sets. We can construct every synchronous set as a union of irreducible synchronous subsets:

Definition 9 (Irreducibility). A non-empty synchronous set $\emptyset \neq S \subseteq R_{0} \cup R_{1}$ is irreducible if and only if $S=S_{0} \cup S_{1}$ implies $S=S_{0}$ or $S=S_{1}$, for all synchronous subsets $S_{0}, S_{1} \subseteq R_{0} \cup R_{1}$.

Example 13. Let $R_{0}$ and $R_{1}$ be sets of rules, and let $\rho \in R_{0}$ be a rule, such that free $(\rho) \cap$ free $\left(R_{1}\right)=\emptyset$. We show that $\{\rho\}$ is irreducible synchronous. Since free $(\{\rho\}) \cap$ free $\left(R_{0}\right)=$ free $(\rho)=$ free $\left(\{\rho\} \cap R_{0}\right)$ and free $(\{\rho\}) \cap$ free $\left(R_{1}\right)=\emptyset \subseteq$ free $\left(\{\rho\} \cap R_{1}\right)$, we conclude that $\{\rho\}$ is synchronous. Suppose $\{\rho\}=S_{0} \cup S_{1}$. Then, $\rho \in S_{i}$, for some $i \in\{0,1\}$. Hence, $\{\rho\} \subseteq S_{i} \subseteq\{\rho\}$, which shows that $S_{i}=\{\rho\}$. We conclude that $\{\rho\}$ is irreducible synchronous in $R_{0} \cup R_{1}$.

Example 14. Consider $\varphi_{i}$ and $\psi_{i}$, for $i \in\{1,2\}$, from Example 12. The irreducible synchronous sets of $\left\{\varphi_{1}, \psi_{1}\right\} \cup\left\{\varphi_{2}, \psi_{2}\right\}$ are $\left\{\varphi_{1}\right\},\left\{\psi_{2}\right\}$, and $\left\{\psi_{1}, \varphi_{2}\right\}$. $\triangle$

Definition 10 (Composition). The composition of sets of rules $R_{0}$ and $R_{1}$ is $R_{0} \wedge R_{1}:=\left\{\bigwedge_{\rho \in S} \rho \mid S \subseteq R_{0} \cup R_{1}\right.$ irreducible synchronous $\}$.

Example 15. Let $R_{0}$ and $R_{1}$ be sets of rules, with free $\left(R_{0}\right) \cap$ free $\left(R_{1}\right)=\emptyset$. By Example 13, we find that $\{\rho\} \subseteq R_{0} \cup R_{1}$, for all $\rho \in R_{0} \cup R_{1}$, is irreducible synchronous. Hence, every synchronous set $S \subseteq R_{0} \cup R_{1}$, with $|S| \geq 2$, is reducible. Therefore, $S \subseteq R_{0} \cup R_{1}$ is irreducible synchronous if and only if $S=\{\rho\}$, for some $\rho \in R_{0} \cup R_{1}$. We conclude that $R_{0} \wedge R_{1}=R_{0} \cup R_{1}$. Consequently, $\emptyset$ is a (unique) identity element with respect to composition $\wedge$ of sets of rules.

To show that the composition of sets of rules coincides with conjunction of stream constraints, we need the following result that shows that every non-empty synchronous set can be covered by irreducible synchronous sets. 
Lemma 3. Let $R_{0}$ and $R_{1}$ be sets of rules, and let $S \subseteq R_{0} \cup R_{1}$ be a non-empty synchronous set. Then, $S=\bigcup_{i=1}^{n} S_{i}$, where $S_{i} \subseteq R_{0} \cup R_{1}$, for $1 \leq i \leq n$, is irreducible synchronous.

Proof. We prove the lemma by induction on the size $|S|$ of $S$. For the base case, suppose that $|S|=1$. We show that $S$ is irreducible synchronous, which provides a trivial covering. Suppose that $S=S_{0} \cup S_{1}$, for some synchronous sets $S_{0}, S_{1} \subseteq R_{0} \cup R_{1}$. Since, $|S|=1$, we have $S \subseteq S_{i} \subseteq S$, for some $i \in\{0,1\}$. Hence, $S=S_{i}$, and $S$ is irreducible. We conclude that the lemma holds, for $|S|=1$.

For the induction step, suppose that $|S|=k>1$, and suppose that the lemma holds, for $|S|<k$. If $S$ is irreducible, we find a trivial covering of $S$. If $S$ is reducible, we find $S=S_{0} \cup S_{1}$, where $S_{0} \neq S \neq S_{1}$ are synchronous sets in $R_{0} \cup R_{1}$. Since $\left|S_{i}\right|<|S|$, for $i \in\{0,1\}$, we find by the hypothesis that $S_{i}=\bigcup_{j=1}^{n_{i}} S_{i j}$. Hence, $S=S_{0} \cup S_{1}=\bigcup_{i=0}^{1} \bigcup_{j=1}^{n_{i}} S_{i j}$. We conclude that the lemma holds, for $|S|=k$. By induction on $|S|$, we conclude the lemma.

Lemma 4. $\operatorname{deg}_{x}(\operatorname{rbf}(R))=\max _{\rho \in R} \operatorname{deg}_{x}(\rho)$, for all sets of rules $R$ and $x \in X$.

Proof. For any set of rules $R$ and $y \in X$, we have

$$
\operatorname{deg}_{y}(\operatorname{rbf}(R))=\max _{x \in \text { free }(R)} \max \left(\operatorname{deg}_{y}\left(x \dagger_{d(x)}\right), \underset{\rho \in R: x \in \operatorname{mree}(\rho)}{\max } \operatorname{deg}_{y}(\rho)\right) .
$$

Note that $\operatorname{deg}_{y}\left(x \dagger_{d(x)}\right)=d(y)$, if $y=x$, and $\operatorname{deg}_{y}\left(x \dagger_{d(x)}\right)=-1$, otherwise. Since $d(y)=\max _{\rho \in R} \operatorname{deg}_{y}(\rho)$, we have $\operatorname{deg}_{y}(\operatorname{rbf}(R))=\max _{\rho \in R} \operatorname{deg}_{y}(\rho)$.

Theorem 3. $\operatorname{rbf}\left(R_{0} \wedge R_{1}\right) \simeq \operatorname{rbf}\left(R_{0}\right) \wedge \operatorname{rbf}\left(R_{1}\right)$, for all sets of rules $R_{0}$ and $R_{1}$.

Proof. By Lemma 4 and Definition 10, $\operatorname{deg}_{x}\left(\operatorname{rbf}\left(R_{0} \wedge R_{1}\right)\right)=\operatorname{deg}_{x}\left(\operatorname{rbf}\left(R_{0}\right) \wedge\right.$ $\left.\operatorname{rbf}\left(R_{1}\right)\right)$, for all $x \in X$. Hence, free ${ }^{k}\left(\operatorname{rbf}\left(R_{0} \wedge R_{1}\right)\right)=\operatorname{free}^{k}\left(\operatorname{rbf}\left(R_{0}\right) \wedge \operatorname{rbf}\left(R_{1}\right)\right)$, for all $k \geq 0$.

Next, we show $\left.\operatorname{rbf}\left(R_{0}\right) \wedge \operatorname{rbf}\left(R_{1}\right)\right) \models \operatorname{rbf}\left(R_{0} \wedge R_{1}\right)$. Let $\theta \in \mathcal{L}\left(\operatorname{rbf}\left(R_{0}\right) \wedge\right.$ $\left.\operatorname{rbf}\left(R_{1}\right)\right)$. By Definition 7 , we must show that for every $x \in \operatorname{free}\left(R_{0} \wedge R_{1}\right)$ there exists some $\rho_{x} \in R_{0} \wedge R_{1}$ such that $x \in$ free $\left(\rho_{x}\right)$ and either $\theta \models x \dagger_{d(x)}$ or $\theta \models \rho_{x}$. Hence, suppose that $\theta \notin \mathcal{L}\left(x \dagger_{d(x)}\right)$, for some variable $x \in$ free $\left(R_{0} \wedge R_{1}\right)$. Since free $\left(R_{0} \wedge R_{1}\right)=$ free $\left(R_{0}\right) \cup$ free $\left(R_{1}\right)$ and $\theta \models$ free $\left(R_{0}\right) \wedge$ free $\left(R_{1}\right)$, we find from Definition 7 some $\psi \in R_{0} \cup R_{1}$, with $\theta \models \psi$ and $x \in$ free $(\psi)$. We now show that there exists an irreducible synchronous set $S \subseteq R_{0} \cup R_{1}$, such that, for $\rho_{x}:=\bigwedge_{\rho \in S} \rho$, we have $\theta \models \rho_{x}$ and $x \in$ free $\left(\rho_{x}\right)$. By repeated application of Definition 8 , we construct a finite sequence

$$
\{\psi\}=S_{0} \subsetneq \cdots \subsetneq S_{n}
$$

such that $S_{n} \subseteq R_{0} \cup R_{1}$ is synchronous, and $\theta=\bigwedge_{\rho \in S_{n}} \rho$. Suppose $S_{k} \subseteq R_{0} \cup R_{1}$, for $k \geq 1$, is not synchronous. By Definition 8 , there exists some $i \in\{0,1\}$ and a variable $x \in$ free $\left(S_{k}\right) \cap$ free $\left(R_{i}\right)$, such that $x \notin$ free $\left(S_{k} \cap R_{i}\right)$. Since $x \in \operatorname{free}\left(R_{i}\right)$, we have $R_{i}^{x}:=\left\{\rho \in R_{i} \mid x \in\right.$ free $\left.(\rho)\right\} \neq \emptyset$. Since $\theta \mid=\operatorname{rbf}\left(R_{i}\right)$, there exists some $\psi_{k} \in R_{i}^{x}$ such that $\theta \models \psi_{k}$. Now define $S_{k+1}:=S_{k} \cup\left\{\psi_{k}\right\}$. Since $x \notin \operatorname{free}\left(S_{k} \cap R_{i}\right)$ 


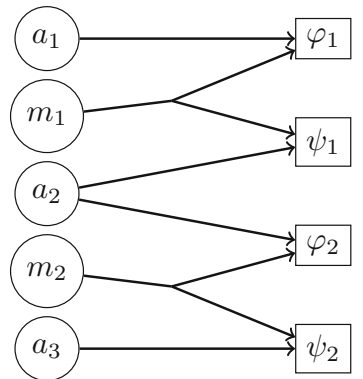

(a) $\bigwedge_{i=1}^{2} \operatorname{rbf}\left(\left\{\varphi_{i}, \psi_{i}\right\}\right)$

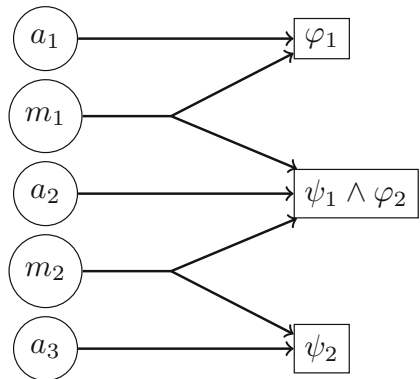

(b) $\operatorname{rbf}\left(\bigwedge_{i=1}^{2}\left\{\varphi_{i}, \psi_{i}\right\}\right)$

Fig. 2. Hypergraph representations of $\bigwedge_{i=1}^{2}$ fifo $\left(a_{i}, a_{i+1}, m_{i}\right)$.

and $x \in \operatorname{free}\left(S_{k+1} \cap R_{i}\right)$, we have a strict inclusion $S_{k} \subsetneq S_{k+1}$. Due to these strict inclusions, we have, for $k \geq\left|R_{0} \cup R_{1}\right|$, that $S_{k}=R_{0} \cup R_{1}$, which is trivially synchronous in $R_{0} \cup R_{1}$. Therefore, our sequence $S_{0} \subsetneq \cdots$ of inclusions terminates, from which we conclude the existence of $S_{n}$. By Lemma 3, we find some irreducible synchronous set $S \subseteq S_{n}$, such that $\psi \in S$. We conclude that $\rho_{x}:=\bigwedge_{\rho \in S} \rho \in R_{0} \wedge R_{1}$ satisfies $\theta \models \rho_{x}$ and $x \in \operatorname{free}(\psi) \subseteq \operatorname{free}(S)=\operatorname{free}\left(\rho_{x}\right)$. By Definition 7 , we have $\theta \models \operatorname{rbf}\left(R_{0} \wedge R_{1}\right)$, and $\operatorname{rbf}\left(R_{0}\right) \wedge \operatorname{rbf}\left(R_{1}\right) \models \operatorname{rbf}\left(R_{0} \wedge R_{1}\right)$.

Finally, we prove that $\operatorname{rbf}\left(R_{0} \wedge R_{1}\right) \models \operatorname{rbf}\left(R_{0}\right) \wedge \operatorname{rbf}\left(R_{1}\right)$. Let $\theta \in \mathcal{L}\left(\operatorname{rbf}\left(R_{0} \wedge\right.\right.$ $\left.R_{1}\right)$ ). We show that $\theta \models \operatorname{rbf}\left(R_{i}\right)$, for all $i \in\{0,1\}$. By Definition 7 , we must show that for every $i \in\{0,1\}$ and every $x \in$ free $\left(R_{i}\right)$ there exists some $\rho \in R_{i}$ such that $x \in$ free $(\rho)$ and either $\theta \models x \dagger_{d(x)}$ or $\theta \models \rho$. Hence, let $i \in\{0,1\}$ and $x \in \operatorname{free}\left(R_{i}\right)$ be arbitrary, and suppose that $\theta \notin \mathcal{L}\left(x \dagger_{d(x)}\right)$. Since free $\left(R_{i}\right) \subseteq \operatorname{free}\left(R_{0} \wedge R_{1}\right)$, it follows from our assumption $\theta \models \operatorname{rbf}\left(R_{0} \wedge R_{1}\right)$ that $\theta \models \bigwedge_{\rho \in S} \rho$, for some irreducible synchronous set $S \subseteq R_{0} \cup R_{1}$ satisfying $x \in$ free $(S)$. Since $S \subseteq R_{0} \cup R_{1}$ synchronous, we find that $x \in \operatorname{free}(S) \cap \operatorname{free}\left(R_{i}\right)=\operatorname{free}\left(S \cap R_{i}\right)$. Hence, we find some $\rho \in S \cap R_{i}$, such that $\theta \models \rho$ and $x \in \operatorname{free}(\rho)$. By Definition 7, we conclude that $\theta \models \operatorname{rbf}\left(R_{i}\right)$, for all $i \in\{0,1\}$. Therefore, $\operatorname{rbf}\left(R_{0} \wedge R_{1}\right) \simeq$ $\operatorname{rbf}\left(R_{0}\right) \wedge \operatorname{rbf}\left(R_{1}\right)$.

Example 16. Let $\varphi_{i}$ and $\psi_{i}$, for $i \geq 1$, be the rules from Example 12. By Example 14 , the composition fifo $_{2}:=\bigwedge_{i=1}^{2}$ fifo $\left(a_{i}, a_{i+1}, m_{i}\right)$ is defined by the set of rules $\left\{\varphi_{1}, \psi_{1} \wedge \varphi_{2}, \psi_{2}\right\} .{ }^{1}$ To compute a set of rules that defines the composition, it is not efficient to enumerate all (exponentially many) synchronous subsets of $R_{0} \cup R_{1}$ and remove all reducible sets. Our tools use an algorithm based on

\footnotetext{
${ }^{1}$ The rules for the composition of two fifo stream constraints has striking similarities with synchronous region decomposition developed by Proença et al. [24]. Indeed, $\varphi_{1}$, $\psi_{1} \wedge \varphi_{2}$, and $\psi_{2}$ correspond to the synchronous regions in the composition of two buffers. Therefore, rule-based composition generalizes synchronous region decomposition that has been used as a basis for generation of parallel code [15].
} 
hypergraph transformations to compute the irreducible synchronous sets. The details of this algorithm fall outside the scope of this paper. Figure 2 shows a graphical representation of composition fifo $_{2}$, using hypergraphs. These hypergraphs consist of sets of hyperedges $(x, F)$, where $x$ is a variable and $F$ is a set of rules. Each hyperedge $(x, F)$ in a hypergraph corresponds to a disjunction $x \dagger_{d(x)} \vee \bigvee_{\rho \in F} \rho$ of the rule-based form in Definition 7.

\section{Complexity}

In the worst case, composition $R_{0} \wedge R_{1}$ of arbitrary sets of rules $R_{0}$ and $R_{1}$ may consists of $\left|R_{0}\right| \times\left|R_{1}\right|$ rules. However, if $R_{0}$ and $R_{1}$ are simple, the size of the composition is bounded by $\left|R_{0}\right|+\left|R_{1}\right|$.

Definition 11 (Simple). A set $R$ of rules is simple if and only if free $(\rho) \cap$ free $\left(\rho^{\prime}\right) \cap P(\operatorname{rbf}(R)) \neq \emptyset$ implies $\rho=\rho^{\prime}$, for every $\rho, \rho^{\prime} \in R$.

Example 17. By Example 10, the invariant of fifo $(a, b, m)$ is defined by $R:=$ $\left\{a \doteq m^{\prime} \doteq \mathbf{0} \wedge m \doteq *, m^{\prime} \doteq * \wedge b \doteq m \doteq \mathbf{0}\right\}$ as well as $R^{\prime}:=\left\{a \doteq m^{\prime} \doteq \mathbf{0} \wedge b \doteq\right.$ $\left.m \doteq *, a \doteq m^{\prime} \doteq * \wedge b \doteq m \doteq \mathbf{0}\right\}$. The set $R$ is simple, while $R^{\prime}$ is not.

Lemma 5. Let $R_{0}$ and $R_{1}$ be sets of rules, such that free $\left(R_{0}\right) \cap$ free $\left(R_{1}\right) \subseteq$ $P\left(\operatorname{rbf}\left(R_{0} \cup R_{1}\right)\right)$, and let $S \subseteq R_{0} \cup R_{1}$ be synchronous. Let $G_{S}$ be a graph with vertices $S$ and edges $E_{S}=\left\{\left(\rho, \rho^{\prime}\right) \in S^{2} \mid\right.$ free $(\rho) \cap$ free $\left.\left(\rho^{\prime}\right) \cap P\left(\operatorname{rbf}\left(R_{0} \cup R_{1}\right)\right) \neq \emptyset\right\}$. If $S$ irreducible, then $G_{S}$ is connected.

Proof. Suppose that $G_{S}$ is disconnected. We find $\emptyset \neq S_{0}, S_{1} \subseteq S$, with $S_{0} \cup S_{1}=$ $S, S_{0} \cap S_{1}=\emptyset$ and free $\left(S_{0}\right) \cap$ free $\left(S_{1}\right) \cap P\left(\operatorname{rbf}\left(R_{0} \cup R_{1}\right)\right)=\emptyset$. We show that $S_{0}$ and $S_{1}$ are synchronous. Let $i, j \in\{0,1\}$ and $x \in$ free $\left(S_{i}\right) \cap$ free $\left(R_{j}\right)$. We distinguish two cases:

Case $1\left(x \in\right.$ free $\left.\left(R_{1-j}\right)\right)$ : Then, $x \in$ free $\left(R_{0}\right) \cap$ free $\left(R_{1}\right) \subseteq P\left(\operatorname{rbf}\left(R_{0} \cup R_{1}\right)\right)$. Since free $\left(S_{0}\right) \cap$ free $\left(S_{1}\right) \cap P\left(\operatorname{rbf}\left(R_{0} \cup R_{1}\right)\right)=\emptyset$, we have $x \notin$ free $\left(S_{1-i}\right)$. Since $S$ is synchronous, we have $x \in$ free $\left(S_{i}\right) \cap$ free $\left(R_{j}\right) \subseteq$ free $(S) \cap$ free $\left(R_{j}\right) \subseteq$ free $\left(S \cap R_{j}\right)$. Hence, we find some $\rho \in S \cap R_{j}$, with $x \in$ free $(\rho)$. Since $x \notin$ free $\left(S_{1-i}\right)$, we conclude that $\rho \in S_{i} \cap R_{j}$. Thus, $x \in$ free $\left(S_{i} \cap R_{j}\right)$, if $x \in \operatorname{free}\left(R_{1-j}\right)$.

Case $2\left(x \notin\right.$ free $\left.\left(R_{1-j}\right)\right)$ : Since $x \in$ free $\left(S_{i}\right)$, we find some $\rho \in S_{i}$, with $x \in$ free $(\rho)$. Since $x \notin$ free $\left(R_{1-j}\right)$, we conclude that $\rho \in R_{j}$. Hence, $x \in$ free $(\rho) \subseteq$ free $\left(S_{i} \cap R_{j}\right)$, if $x \notin$ free $\left(R_{1-j}\right)$.

We conclude in both cases that $x \in$ free $(\rho) \subseteq$ free $\left(S_{i} \cap R_{j}\right)$. Hence, free $\left(S_{i}\right) \cap$ free $\left(R_{j}\right) \subseteq$ free $\left(S_{i} \cap R_{j}\right)$, for all $i, j \in\{0,1\}$, and we conclude that $S_{0}$ and $S_{1}$ are synchronous. Since $S_{0} \neq S \neq S_{1}$, we conclude that $S$ is reducible. By contraposition, we conclude that $G_{S}$ is connected, whenever $S$ is irreducible.

Lemma 6. Let $R_{0}$ and $R_{1}$ be simple sets of rules, with free $\left(R_{0}\right) \cap$ free $\left(R_{1}\right) \subseteq$ $P\left(\operatorname{rbf}\left(R_{0} \cup R_{1}\right)\right)$, and let $S_{0}, S_{1} \subseteq R_{0} \cup R_{1}$ be irreducible synchronous. If $S_{0} \cap S_{1} \neq$ $\emptyset$, then $S_{0}=S_{1}$. 
Proof. Suppose that $S_{0} \cap S_{1} \neq \emptyset$. Then, there exists some $\rho_{0} \in S_{0} \cap S_{1}$. We show that $S_{i} \subseteq S_{1-i}$, for all $i \in\{0,1\}$. Let $i \in\{0,1\}$, and $\rho \in S_{i}$. By Lemma 5 , we find an undirected path in $G_{S_{i}}$ from $\rho_{0}$ to $\rho$. That is, we find a sequence $\rho_{0} \rho_{1} \cdots \rho_{n} \in S^{*}$, such that $\rho_{n}=\rho$ and $\left(\rho_{i}, \rho_{i+1}\right) \in E_{S_{i}}$, for all $0 \leq i<n$. We show by induction on $n \geq 0$, that $\rho_{n} \in S_{1-i}$. For the base case $(n=0)$, observe that $\rho_{n}=\rho_{0} \in S_{0} \cap S_{1} \subseteq S_{1-i}$. For the induction step, suppose that $\rho_{n} \in S_{1-i}$. By construction of $G_{S_{i}}$, we find that free $\left(\rho_{n}\right) \cap$ free $\left(\rho_{n+1}\right) \cap P_{01} \neq \emptyset$, where $P_{01}=$ $P\left(\operatorname{rbf}\left(R_{0} \cup R_{1}\right)\right)$. Let $j \in\{0,1\}$, such that $\rho_{n+1} \in R_{j}$. Since $\rho_{n} \in S_{1-i}$ and $S_{1-i}$ is synchronous, we have $\emptyset \neq \operatorname{free}\left(S_{1-i}\right) \cap \operatorname{free}\left(R_{j}\right) \cap P_{01}=\operatorname{free}\left(S_{1-i} \cap R_{j}\right) \cap P_{01}$. We find some $\rho^{\prime} \in S_{1-j} \cap R_{j}$, with free $\left(\rho_{n+1}\right) \cap$ free $\left(\rho^{\prime}\right) \cap P_{01} \neq \emptyset$. Since $R_{j}$ is simple, we have $\rho_{n+1}=\rho^{\prime} \in S_{1-i}$, which concludes the proof by induction. It follows from $\rho_{n} \in S_{1-i}$ that $S_{i} \subseteq S_{1-i}$, for all $i \in\{0,1\}$, that is, $S_{0}=S_{1}$.

As seen in Lemma 2, the number of clauses in the disjunctive normal form $\operatorname{dnf}\left(R_{0} \wedge R_{1}\right)$ can be exponential in the number of rules $\left|R_{0} \wedge R_{1}\right|$ of the composition of $R_{0}$ and $R_{1}$. However, the following (main) theorem shows the number of rules required to define $\bigwedge_{i} \phi_{i}$ is only linear in $k$.

Theorem 4. If $R_{0}$ and $R_{1}$ are simple sets of rules, and free $\left(R_{0}\right) \cap \operatorname{free}\left(R_{1}\right) \subseteq$ $P\left(\operatorname{rbf}\left(R_{0} \cup R_{1}\right)\right)$, then $R_{0} \wedge R_{1}$ is simple and $\left|R_{0} \wedge R_{1}\right| \leq\left|R_{0}\right|+\left|R_{1}\right|$.

Proof. From Lemmas 3 and 6, we find that the irreducible synchronous subsets partition $R_{0} \cup R_{1}$. We conclude that $\left|R_{0} \wedge R_{1}\right| \leq\left|R_{0}\right|+\left|R_{1}\right|$. We now show that $R_{0} \wedge R_{1}$ is simple. Let $\rho_{0}$ and $\rho_{1}$ be rules in $R_{0} \wedge R_{1}$, with free $\left(\rho_{0}\right) \cap$ free $\left(\rho_{1}\right) \cap P_{01} \neq \emptyset$, where $P_{01}=P\left(\operatorname{rbf}\left(R_{0} \cup R_{1}\right)\right)$. By Definition 10, we find, for all $i \in\{0,1\}$, an irreducible synchronous set $S_{i}$, such that $\rho_{i}=\bigwedge_{\psi \in S_{i}} \psi$. Since free $\left(\rho_{0}\right) \cap \operatorname{free}\left(\rho_{1}\right) \cap P_{01} \neq \emptyset$ and free $\left(\rho_{i}\right)=\operatorname{free}\left(S_{i}\right)$, for all $i \in\{0,1\}$, we find some $x \in$ free $\left(S_{0}\right) \cap$ free $\left(S_{1}\right) \cap P_{01}$. Suppose that $x \in$ free $\left(R_{j}\right)$, for some $j \in\{0,1\}$. Since $S_{0}$ and $S_{1}$ are synchronous sets, we have $x \in \operatorname{free}\left(S_{i}\right) \cap \operatorname{free}\left(R_{j}\right) \subseteq \operatorname{free}\left(S_{i} \cap R_{j}\right)$, for all $i \in\{0,1\}$. We find, for all $i \in\{0,1\}$, some $\psi_{i} \in S_{i} \cap R_{j}$, such that $x \in \operatorname{free}\left(\psi_{i}\right)$. Hence, free $\left(\psi_{0}\right) \cap$ free $\left(\psi_{1}\right) \cap P_{01} \neq \emptyset$, and since $R_{j}$ is simple, we conclude that $\psi_{0}=\psi_{1}$. Therefore, $S_{0} \cap S_{1} \neq \emptyset$, and Lemma 6 shows that $S_{0}=S_{1}$ and $\rho_{0}=\rho_{1}$. We conclude that $R_{0} \wedge R_{1}$ is simple.

The number of clauses in the disjunctive normal form of direct compositions of $k$ fifo constraints grows exponentially in $k$. This typical pattern of a sequence of queues manifests itself in many other constructions, which causes serious scalability problems (cf., the benchmarks for 'Alternator ${ }_{k}$ ' in [17, Sect. 7.2]). However, Theorem 4 shows that rule-based composition of $k$ fifo constraints does not suffer from scalability issues: by Example 17, the fifo constraint can be defined by a simple set of rules. The result in Theorem 4, therefore, promises (exponential) improvement over the classical constraint automaton representation.

Unfortunately, it seems impossible to define any arbitrary stream constraint by a simple set of rules. Therefore, the rule-based form may still blow up for certain stream constraints. It seems, however, possible to recognize even more symmetries (cf., the queue-optimization in [16]) to avoid explosion and obtain comparable compilation and execution performance for these stream constraints. 


\section{Abstraction}

We now study how existential quantification of stream constraints operates on its defining set of rules.

Definition 12 (Abstraction). Hiding a variable $x$ in a set of rules $R$ yields $\exists x R:=\{\exists x \rho \mid \rho \in R\}$.

Unfortunately, $\exists x R$ does not always define $\exists x \phi$, for a stream constraint $\phi$ defined by a set of rules $R$. The following result shows that $\exists x R$ defines $\exists x \phi$ if and only if $\operatorname{rbf}(\exists x R)=\exists x \operatorname{rbf}(R)$. In this case, we call variable $x$ hidable in $R$.

It is non-trivial to find a defining set of rules for $\exists x \phi$, if $x$ is not hidable in $R$, and we leave this as future work.

Theorem 5. Let $R$ be a set of rules, and let $x \in X$ be a variable. Then, $\exists x \operatorname{rbf}(R) \simeq \operatorname{rbf}(\exists x R)$ if and only if $\operatorname{rbf}(\exists x R) \models \exists x \operatorname{rbf}(R)$.

Proof. Trivially, $\exists x \operatorname{rbf}(R) \simeq \operatorname{rbf}(\exists x R)$ implies $\operatorname{rbf}(\exists x R) \models \exists x \operatorname{rbf}(R)$. Conversely, suppose that $\operatorname{rbf}(\exists x R) \models \exists x \operatorname{rbf}(R)$. From Lemma 2, it follows that $\exists x \operatorname{rbf}(R) \equiv \exists x \operatorname{dnf}(R)$. Since existential quantification distributes over disjunction and $\exists x \phi \wedge \psi \models \exists x \phi \wedge \exists x \psi$, for all stream constraints $\phi$ and $\psi$, we find

$$
\exists x \operatorname{dnf}(R) \models \bigvee_{S \subseteq R} \bigwedge_{\rho \in S} \exists x \rho \wedge \bigwedge_{x \neq y \in \operatorname{free}(R) \backslash \operatorname{free}(S)} y \dagger^{d} d(y) \equiv \operatorname{dnf}(\exists x R) .
$$

By Lemma 2, we have $\exists x \operatorname{rbf}(R) \models \operatorname{rbf}(\exists x R)$, and by assumption $\exists x \operatorname{rbf}(R) \equiv$ $\operatorname{rbf}(\exists x R)$. Using Lemma 4, we have $\operatorname{deg}_{y}(\exists x \operatorname{rbf}(R))=\max _{\rho \in R} \operatorname{deg}_{y}(\exists x \rho)=$ $\operatorname{deg}_{y}(\operatorname{rbf}(\exists x R))$, for every variable $y$. We conclude $\exists x \operatorname{rbf}(R) \simeq \operatorname{rbf}(\exists x R)$.

Example 18. Suppose Data $=\{0,1\}$, which means that the data domain equals $D=\{0,1, *\}$. Let $\mathbf{1}$ be the constant stream defined as $\mathbf{1}(n)=1$, for all $n \in \mathbb{N}$. For $i \in\{0,1\}$, consider the set of rules $R_{i}=\left\{x=x, x=y_{i}=\mathbf{i}\right\}$. Observe that $\left\{x=x, x=y_{i}=\mathbf{i}\right\} \subseteq R_{0} \cup R_{1}$ is synchronous, for all $i \in\{0,1\}$. Hence, $x=y_{i}=\mathbf{i} \in R_{0} \wedge R_{1}$, for all $i \in\{0,1\}$. However, for $\theta=\left[y_{0} \mapsto \mathbf{0}, y_{1} \mapsto \mathbf{1}\right]$, we have $\theta \models \bigwedge_{i \in\{0,1\}} \exists x\left(x=y_{i}=i\right)$, while $\exists x \bigwedge_{i \in\{0,1\}} x=y_{i}=i \equiv \perp$. Thus, variable $x$ is not hidable from $R_{0} \wedge R_{1}$.

\section{Application}

In on-going work, we applied the rule-based form to compile protocols (in the form of Reo connectors) into executable code. Reo is an exogenous coordination language that models protocols as graph-like structures $[1,2]$. We recently developed a textual version of Reo, which we use to design non-trivial protocols [11]. An example of such non-trivial protocol is the Alternator ${ }_{k}$, where $k \geq 2$ is an integer. Figure 3(a) shows a graphical representation of the Alternator protocol.

Intuitively, the behavior of the alternator protocol is as follows: The nodes $P_{1}, \ldots, P_{k}$ accept data from the environment. Node $C$ offer data to the environment. All other nodes are internal and do not interact with the environment. 


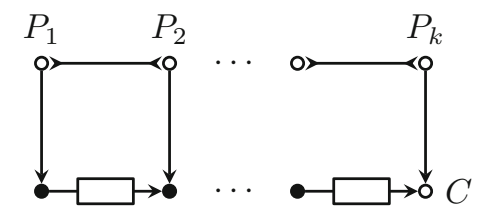

(a) Alternator $k$

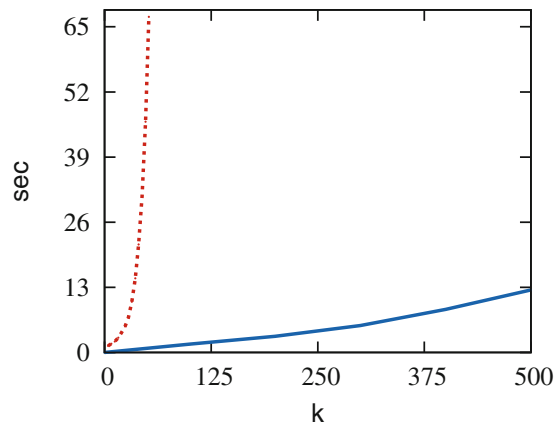

(b) Compilation times

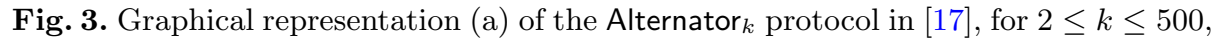
and its compilation time (b). The dotted red line is produced by the Jongmans' compiler (and corresponds to [17, Fig. 11(a)]), and the solid blue line is our compiler. (Color figure online)

In the first step of the protocol, the Alternator $k$ waits until the environment is ready to offer data at all nodes $P_{1}, \ldots, P_{k}$ and is ready to accept data from node $C$. Only then, the Alternator ${ }_{k}$ transfers the data from $P_{k}$ to $C$ via a synchronous channel, and puts the data from $P_{i}$ in the $i$-th fifo channel, for all $i<k$. The behavior of a synchronous channel is defined by the sync stream constraint in Example 1. Each fifo channel has buffer capacity of one, and its behavior is defined by the fifo stream constraint from Example 3. In subsequent steps, the environment can one-by-one retrieve the data from the fifo channel buffers, until they are all empty. Then, the protocol cycles back to its initial configuration, and repeats its behavior. For more details on the Reo language and its semantics, we refer to $[1,2]$.

As mentioned in the introduction, Jongmans developed a compiler based on constraint automata [17]. The otherwise stimulating benchmarks presented in [17] show that Jongmans' compiler still suffers from state-space explosion.

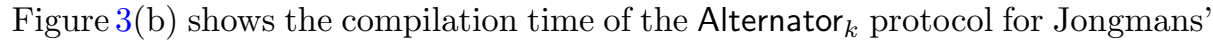
compiler and ours. Clearly, the compilation time improved drastically and went from exponential in $k$ to almost linear in $k$.

Every fifo channel in the Alternator ${ }_{k}$, except the first, either accepts data from the environment or accepts data from the previous fifo channel. This choice is made by the internal node at the input of each fifo channel. Unfortunately, the behavior of such nodes is not defined in terms of a simple set of rules. Consequently, we cannot readily apply Theorem 4 to conclude that the number of rules depends only linearly on $k$. However, it turns out that Alternator ${ }_{k}$ can be defined using only $k$ rules: one rule for filling the buffers of all fifo channels, plus $k-1$ rules, one for taking data out of the buffer of each of the $k-1$ fifo channels. This observation explains why our compiler drastically improves upon Jongmans' compiler. 


\section{Conclusion}

We introduce (regular) stream constraints as an alternative to constraint automata that does not suffer from state space explosions. We define the rulebased form for stream constraints, and we express composition and abstraction of constraints in terms of their rule-based forms. For simple sets of rules, composition of rule-based forms does not suffer from 'transition space explosions' either.

We have experimented with a new compiler for protocols using our rulebased form, which avoids the scalability problems of state- and transition-space explosions of previous automata-based tools. Our approach still leaves the possibility for transition space explosion for non-simple sets of rules. In the future, we intend to study symmetries in stream constraints that are not defined by simple sets of rules. The queue-optimization of Jongmans serves as a good source of inspiration for exploiting symmetries [16].

The results in this paper are purely theoretical. In on-going work, we show practical implications of our results by developing a compiler based on stream constraints. Such a compiler requires an extension to the current theory on stream constraints: we did not compute the abstraction $\exists x R$ on sets of rules $R$ wherein variable $x$ is not hidable. Example 11 indicates the existence of situations where we can compute $\exists x R$ even if $x$ is not hidable, a topic which we leave as future work.

Acknowledgements. The authors thank Benjamin Lion for his help in developing a rule-based compiler and for generating Fig. 3(b).

\section{References}

1. Arbab, F.: Reo: a channel-based coordination model for component composition. Math. Struct. Comput. Sci. 14(3), 329-366 (2004). https://doi.org/10.1017/ S0960129504004153

2. Arbab, F.: Puff, the magic protocol. In: Agha, G., Danvy, O., Meseguer, J. (eds.) Formal Modeling: Actors, Open Systems, Biological Systems. LNCS, vol. 7000, pp. 169-206. Springer, Heidelberg (2011). https://doi.org/10.1007/978-3-642-249334_9

3. Arbab, F., Rutten, J.J.M.M.: A coinductive calculus of component connectors. In: Wirsing, M., Pattinson, D., Hennicker, R. (eds.) WADT 2002. LNCS, vol. 2755, pp. 34-55. Springer, Heidelberg (2003). https://doi.org/10.1007/978-3-540-40020$2 \_2$

4. Baier, C., Blechmann, T., Klein, J., Klüppelholz, S.: A uniform framework for modeling and verifying components and connectors. In: Field, J., Vasconcelos, V.T. (eds.) COORDINATION 2009. LNCS, vol. 5521, pp. 247-267. Springer, Heidelberg (2009). https://doi.org/10.1007/978-3-642-02053-7_13

5. Baier, C., Clarke, E.M., Hartonas-Garmhausen, V., Kwiatkowska, M., Ryan, M.: Symbolic model checking for probabilistic processes. In: Degano, P., Gorrieri, R., Marchetti-Spaccamela, A. (eds.) ICALP 1997. LNCS, vol. 1256, pp. 430-440. Springer, Heidelberg (1997). https://doi.org/10.1007/3-540-63165-8_199 
6. Baier, C., Sirjani, M., Arbab, F., Rutten, J.J.M.M.: Modeling component connectors in Reo by constraint automata. Sci. Comput. Program. 61(2), 75-113 (2006). https://doi.org/10.1016/j.scico.2005.10.008

7. Bryant, R.E.: Graph-based algorithms for Boolean function manipulation. IEEE Trans. Comput. 35(8), 677-691 (1986). https://doi.org/10.1109/TC.1986.1676819

8. Burch, J.R., Clarke, E.M., McMillan, K.L., Dill, D.L., Hwang, L.J.: Symbolic model checking: $10^{\wedge} 20$ states and beyond. Inf. Comput. 98(2), 142-170 (1992). https:// doi.org/10.1016/0890-5401(92)90017-A

9. Clarke, D., Proença, J., Lazovik, A., Arbab, F.: Channel-based coordination via constraint satisfaction. Sci. Comput. Program. 76(8), 681-710 (2011). https://doi. org/10.1016/j.scico.2010.05.004

10. Clavel, M., Durán, F., Eker, S., Lincoln, P., Martí-Oliet, N., Meseguer, J., Talcott, C.L. (eds.): All About Maude - A High-Performance Logical Framework. LNCS, vol. 4350. Springer, Heidelberg (2007). https://doi.org/10.1007/978-3-540-719991

11. Dokter, K., Arbab, F.: Treo: textual syntax of reo connectors. In: Proceedings of MeTRiD 2018 (2018, to appear)

12. Ehlers, R.: Minimising deterministic Büchi automata precisely using SAT solving. In: Strichman, O., Szeider, S. (eds.) SAT 2010. LNCS, vol. 6175, pp. 326-332. Springer, Heidelberg (2010). https://doi.org/10.1007/978-3-642-14186-7_28

13. Jongmans, S.T.Q.: Automata-theoretic protocol programming. Ph.D. thesis, Centrum Wiskunde \& Informatica (CWI), Faculty of Science, Leiden University (2016). http://hdl.handle.net/1887/38223

14. Jongmans, S.-S.T.Q., Arbab, F.: Take Command of Your Constraints! In: Proceedings of Coordination Models and Languages - 17th IFIP WG 6.1 International Conference, COORDINATION 2015, Held as Part of the 10th International Federated Conference on Distributed Computing Techniques, DisCoTec 2015, Grenoble, France, June 2-4, 2015, pp. 117-132 (2015). https://doi.org/10.1007/978-3-31919282-6_8

15. Jongmans, S.T.Q., Arbab, F.: Centralized coordination vs. partially-distributed coordination with Reo and constraint automata. Sci. Comput. Program. (2017). http://www.sciencedirect.com/science/article/pii/S0167642317301259

16. Jongmans, S.-S.T.Q., Halle, S., Arbab, F.: Automata-based optimization of interaction protocols for scalable multicore platforms. In: Kühn, E., Pugliese, R. (eds.) COORDINATION 2014. LNCS, vol. 8459, pp. 65-82. Springer, Heidelberg (2014). https://doi.org/10.1007/978-3-662-43376-8_5

17. Jongmans, S.T.Q., Kappé, T., Arbab, F.: Constraint automata with memory cells and their composition. Sci. Comput. Program. 146, 50-86 (2017). https://doi.org/ 10.1016/j.scico.2017.03.006

18. Kemper, S.: SAT-based verification for timed component connectors. Sci. Comput. Program. 77(7-8), 779-798 (2012). https://doi.org/10.1016/j.scico.2011.02.003

19. Klüppelholz, S.: Verification of Branching-Time and Alternating-Time Properties for Exogenous Coordination Models. Ph.D. thesis, Dresden University of Technology (2012). http://www.qucosa.de/recherche/frontdoor/?tx slubopus4frontend $[\mathrm{id}]=8621$

20. Kwiatkowska, M.Z., Norman, G., Sproston, J., Wang, F.: Symbolic model checking for probabilistic timed automata. Inf. Comput. 205(7), 1027-1077 (2007). https:// doi.org/10.1016/j.ic.2007.01.004

21. Murata, T.: Petri nets: properties, analysis and applications. Proc. IEEE 77(4), 541-580 (1989) 
22. Pnueli, A., Rosner, R.: On the synthesis of a reactive module. In: Conference Record of the Sixteenth Annual ACM Symposium on Principles of Programming Languages, Austin, Texas, USA, 11-13 January 1989, pp. 179-190. ACM Press (1989). http://doi.acm.org/10.1145/75277.75293

23. Pnueli, A., Rosner, R.: Distributed reactive systems are hard to synthesize. In: 31st Annual Symposium on Foundations of Computer Science, St. Louis, Missouri, USA, 22-24 October 1990, vol. II, pp. 746-757. IEEE Computer Society (1990). https://doi.org/10.1109/FSCS.1990.89597

24. Proença, J., Clarke, D., de Vink, E.P., Arbab, F.: Dreams: a framework for distributed synchronous coordination. In: Ossowski, S., Lecca, P. (eds.) Proceedings of the ACM Symposium on Applied Computing, SAC 2012, Riva, Trento, Italy, 2630 March 2012, pp. 1510-1515. ACM (2012). http://doi.acm.org/10.1145/2245276. 2232017

25. Rutten, J.J.M.M.: Elements of stream calculus (an extensive exercise in coinduction). Electr. Notes Theor. Comput. Sci. 45, 358-423 (2001). https://doi.org/10. 1016/S1571-0661(04)80972-1 